\title{
A NOTE ON MONOTONIC ORTHO-BASES
}

\author{
THOMAS M. PHILLIPS
}

\begin{abstract}
At the 1974 Topology Conference at Charlotte, North Carolina, Peter Nyikos introduced the concept of an ortho-base and announced that a $T_{2}$ paracompact first-countable $\beta$-space having an ortho-base is metrizable. The purpose of this paper is to introduce an obvious monotonic generalization of ortho-bases and to prove the following theorem.

THEOREM. If $S$ is a regular $T_{0}$ space having a monotonic ortho-base, then each of the following implies that $S$ has a base of countable order:

(1) $S$ is connected;

(2) $S$ is a $\beta_{c}$-space;

(3) $S$ is a first-countable monotonic $\beta$-space.

Nyikos' theorem is a corollary to (3) and Arhangel'skil's theorem that a $T_{2}$ paracompact space having a base of countable order is metrizable.
\end{abstract}

An ortho-base for a space $X$ is a base $B$ for the topology of $X$ such that if $F \subseteq B$, then either $\cap F$ is open or $\cap F=\{x\}$ and $F$ is a local base at $x$. Every space having an ortho-base is orthocompact ${ }^{1}$ and for $T_{0}$ developable spaces, the converse is true. However, the space of countable ordinals is an orthocompact space which does not have an ortho-base. The concept of an ortho-base was introduced by Nyikos in [5] where he announced [5, Theorem 3.1] that a $T_{2}$ paracompact first countable $\beta$-space ${ }^{2}$ having an ortho-base is metrizable. The purpose of this note is to show that a regular $T_{0}$ first countable monotonic $\beta$-space having a monotonic ortho-base (see definitions below) has a base of countable order thereby obtaining Nyikos' theorem as a corollary to the well-known theorem of Arhangel'skii that a $T_{2}$ paracompact space having a base of countable order is metrizable.

The primitive concepts of Wicke and Worrell are essential tools in the investigation of spaces having bases of countable order and it is assumed that the reader is acquainted with the terminology and techniques found in [7] and [8]. An example of the utility of these technical results is the following lemma, the proof of which is implicit in the works of these authors.

Presented to the Society, September 12, 1975; received by the editors September 29, 1975 and, in revised form, November $1,1976$.

AMS (MOS) subject classifications (1970). Primary 54E99.

Key words and phrases. Base of countable order, $\beta$-space, monotonic $\beta$-space, monotonic ortho-base, monotonically developable, ortho-base.

${ }^{1}$ A space $X$ is orthocompact [3] if every open cover $G$ of $X$ has an open refinement $H$ such that the intersection of every subcollection of $H$ is open.

${ }^{2} A$ space $X$ is a $\beta$-space [4] if for each $x \in X$, there is a sequence $\left\{g_{n}(x)\right\}_{n=1}^{\infty}$ of open neighborhoods of $x$ such that if $x \in g_{n}\left(x_{n}\right)$ for each $n$, then the sequence $\left\{x_{n}\right\}_{n=1}^{\infty}$ has a cluster point. 
LEMMA. $A$ regular $T_{0}$ space $X$ has a base of countable order if and only if there is a sequence $W=\left(W_{n}\right)$ having the following properties:

(1) $W_{n}$ is a well-ordered open cover of $X$;

(2) each $U \in W_{n}$ contains a point of $X$ not in any predecessor of $U$ in $W_{n}$;

(3) if $x \in X, j<n$, and $U$ and $V$ are the first elements of $W_{j}$ and $W_{n}$, respectively, that contain $x$, then $\bar{V} \subseteq U$;

(4) if (a) $w=\left(w_{n}\right)$ is a sequence such that for each $n$, there is an $x \in X$ such that $w_{i}$ is the first element of $W_{i}$ that contains $x$ for $i \leqslant n+1$ and (b) $P \in \cap_{n=1}^{\infty} w_{n}$, then $\left\{w_{n} \mid n \in N\right\}$ is a local base at $P$.

REMARK. In general a sequence $W$ having properties (1)-(3) in the preceding lemma is called a primitive closurewise sequence of $X$ while a sequence $w$ having property (4)(a) is called a primitive representative of $W$. The adjective "closurewise" may be deleted by omitting the closure symbol in (3).

Definition [2]. A space $X$ is said to be a monotonic $\beta$-space if, for each point $x \in X$, there exists a decreasing sequence $\left\{B_{n}(x)\right\}_{n=1}^{\infty}$ of bases of $X$ at $x$ such that if $b_{n} \in B_{n}\left(x_{n}\right), b_{n+1} \subseteq b_{n}$ and $\bigcap_{n=1}^{\infty} b_{n} \neq \varnothing$, then the sequence $\left\{x_{n}\right\}_{n=1}^{\infty}$ has a cluster point. The family $\left\{\left\{B_{n}(x)\right\}_{n=1}^{\infty} \mid x \in X\right\}$ will be called a monotonic $\beta$-system of $X$. Every essentially $T_{1}$ space having a base of countable order is a monotonic $\beta$-space. Thus the space $X$ in [1, Example 2] is a monotonic $\beta$-space but not a $\beta$-space.

Definition. A monotonic ortho-base for a space $X$ is a base $B$ for the topology of $X$ such that if $F$ is a monotonic subcollection of $B$, then either $\cap F$ is open or $\cap F=\{x\}$ and $F$ is a local base at $x$. For $T_{0}$ spaces, every base of countable order is a monotonic ortho-base. Thus the space of countable ordinals and the space described in [3, Example 4.2] have monotonic ortho-bases but not ortho-bases.

Notation. In the following theorems, $N$ denotes the set of positive integers and $(S, \tau)$ denotes a regular $T_{0}$ space. As in [2], the statement that $S$ is monotonically developable means $S$ has a base of countable order. If $K$ is a well-ordered collection of subsets of $S$ and $u$ is a subset of some element of $K$, then $K(u)$ denotes the first element of $K$ which contains $u$. If $u=\{P\}$, then $K(u)$ is denoted by $K(P)$.

THEOREM. 1. If $S$ is a connected space having a monotonic ortho-base, then $S$ is monotonically developable.

Proof. Let $B$ be a monotonic ortho-base for $S$ and let $W$ be a primitive closurewise sequence of $S$ such that $W_{n} \subseteq B$ for each $n$. Let $w$ be a primitive representative of $W$ and suppose $P \in \cap_{n=1}^{\infty} w_{n}$. If $\left\{w_{n} \mid n \in N\right\}$ is not a local base at $P$, then $\bigcap_{n=1}^{\infty} w_{n}=\bigcap_{n=1}^{\infty} \bar{w}_{n}$ is both open and closed which is a contradiction. So $S$ is monotonically developable by the lemma.

REMARK. Note that Theorem 3.2 of [5] is a corollary to the preceding theorem and Arhangel'skiï's theorem.

Definition [6]. A space $X$ is a $\beta_{c}$-space if there is a sequence $G=\left(G_{n}\right)$ such that 
(1) $G_{n}$ is an open cover of $X$;

(2) for each $n$, if $x \in A \in G_{n}$, there is a $B \in G_{n+1}$ such that $x \in B \subseteq A$;

(3) if for each $n, g_{n} \in G_{n}$ and $g_{n+1} \subseteq g_{n}$, then if $B=\bigcap\left\{\bar{g}_{n} \mid n \in N\right\}$ is nonempty, it is countably compact and $U$ open and $B \subseteq U$ implies that some $g_{k} \subseteq U$.

REMARK. In [7] a sequence $G$ as in the preceding definition is called a $\beta_{c}$-sequence. As observed in [8] for regular spaces, the class of $\beta_{c}$-spaces includes all $p$-spaces, $w \Delta$-spaces, $M$-spaces and quasi-complete spaces.

THEOREM 2. If $S$ is a $\beta_{c}$-space having a monotonic ortho-base, then $S$ is monotonically developable.

Proof. Let $B$ be a monotonic ortho-base for $S$ and let $G$ be a $\beta_{c}$-sequence of $S$. As indicated in [7], we may assume $G$ to be a primitive sequence. Using the techniques employed in the proofs of [9, Lemmas 2.1 and 2.4], a primitive sequence $W$ of $S$ can be constructed so that (1) $W_{n} \subseteq B$ for each $n$; (2) if $P \in S$ and $W_{n}(P)$ is not singleton, then $\overline{W_{n+1}(P)}$ is a proper subset of $W_{n}(P)$; and (3) $W$ is controlled (see [7]) by $G$. By [7, Lemma 7.6 and Remark 7.7l it follows that $W$ is also a primitive $\beta_{c}$-sequence of $S$. Let $w$ be a primitive representative of $W$ and suppose $P \in \cap_{n=1}^{\infty} w_{n}$. Suppose $\left\{w_{n} \mid n \in N\right\}$ is not a local base at $P$. Then for each $n$, there is a point $x_{n}$ in $w_{n}-\bar{w}_{n+1}$. As observed in [6, Remark 5.1], $\left\{x_{n} \mid n \in N\right\}$ has a limit point in $\bigcap_{n=1}^{\infty} \bar{w}_{n}=\bigcap_{n=1}^{\infty} w_{n}$. Thus $\cap_{n=1}^{\infty} w_{n}$ is not open, which is a contradiction. So $\left\{w_{n} \mid n \in N\right\}$ is a local base at $P$ and $S$ is monotonically developable by the lemma.

Definition [8]. A space $X$ has a primitive base if and only if there is a sequence $W=\left(W_{n}\right)$ of well-ordered collections of open sets such that for each $x \in X$, if $U$ is open and $x \in U$, there exist $n$ and $k$ such that $x$ is in $n$ elements of $W_{k}$ and the $n$th such element is a subset of $U$.

THEOREM 3. If $S$ is a first countable monotonic $\beta$-space having a monotonic ortho-base, then $S$ is monotonically developable.

Proof. We will show that $S$ has a primitive base which, as observed in [2], is equivalent to possessing a $(d)$-sequence of ordered covers [2]. By [2, Corollary 2.4], it follows that $S$ has a base of countable order.

Let $\left\{\left\{B_{n}(x)\right\}_{n=1}^{\infty} \mid x \in S\right\}$ be a monotonic $\beta$-system of $S$ such that $\left\{\cup B_{n}(x) \mid n \in N\right\}$ is a decreasing local base at $x$ for each $x \in S$. Let $B$ be a monotonic ortho-base for $S$ and well-order $B$. Well-order the set $W_{1}=\{b \in$ $B \mid b$ is contained in some set in $B_{1}(x)$ for some $\left.x \in b\right\}$ and for each $w \in W_{1}$, choose a point $x(w)$ and a set $b_{1}(w)$ so that $x(w) \in w, w \subseteq b_{1}(w)$, and $b_{1}(w) \in B_{1}(x(w))$.

Suppose $W_{1}, \ldots, W_{n}$ have been defined so that

$\left(\mathrm{I}_{1}\right)$ for each $i \leqslant n, W_{i}$ is a well-ordered subcollection of $B$ covering $S$ such that if $w \in W_{i}$, then a point $x(w)$ and a set $b_{i}(w)$ have been chosen so that $x(w) \in w, w \subseteq b_{i}(w)$, and $b_{i}(w) \in B_{i}(x(w))$; and

$\left(\mathrm{I}_{2}\right)$ if $i \leqslant n-1$ and $P \in S$ such that $P \neq x\left(W_{i}(P)\right)$, then $\overline{W_{i+1}(P)} \subseteq$ 
$W_{i}(P), x\left(W_{i}(P)\right) \notin \overline{W_{i+1}(P)}$, and $b_{i+1}\left(W_{i+1}(P)\right) \subseteq b_{i}\left(W_{i}(P)\right)$.

Let $M=\left\{P \in S \mid P \neq x\left(W_{n}(P)\right)\right\}$ and let $V=\left\{b \in B \mid\right.$ some set in $W_{n}$ contains $\bar{b}, x\left(W_{n}(\bar{b})\right) \notin b$, and there is a point $x \in b$ such that some set in $B_{n+1}(x)$ contains $b$ and is contained in $\left.b_{n}\left(W_{n}(\bar{b})\right)\right\}$. Well-order $V$ so that $u<v$ in $V$ if and only if (1) $W_{n}(\bar{u})<W_{n}(\bar{v})$ or (2) $W_{n}(\bar{u})=W_{n}(\bar{v})$ and $u \leqslant v$ in $B . V$ is a base for $S$ on $M$ and $W_{n}(P)=W_{n}(\overline{V(P)})$ for each $P \in M$. Let $Z=\left\{b \in B \mid b \notin V\right.$ and $b$ is contained in some set in $B_{n+1}(x)$ for some $x \in b\}$ and well-order $W_{n+1}=V \cup Z$ so that the order of $V$ is preserved and every element of $V$ precedes every element of $Z$. For each $w \in W_{n+1}$, choose a point $x(w)$ and a set $b_{n+1}(w)$ so that $x(w) \in w, w \subseteq$ $b_{n+1}(w), b_{n+1}(w) \in B_{n+1}(x(w))$, and if $w \in V$, then $b_{n+1}(w) \subseteq b_{n}\left(W_{n}(\bar{w})\right)$. If $P \in M$, then $W_{n+1}(P)=V(P)$, hence $W_{n}(P)=W_{n}\left(\overline{W_{n+1}(P)}\right)$. Thus

$$
x\left(W_{n}(P)\right)=x\left(W_{n}\left(\overline{W_{n+1}(P)}\right)\right) \notin \overline{W_{n+1}(P)}
$$

and

$$
b_{n+1}\left(W_{n+1}(P)\right) \subseteq b_{n}\left(W_{n}\left(\overline{W_{n+1}(P)}\right)\right)=b_{n}\left(W_{n}(P)\right) .
$$

So $\left(\mathrm{I}_{1}\right)$ and $\left(\mathrm{I}_{2}\right)$ are satisfied when $n$ is replaced by $n+1$. Consequently there is a sequence $W=W_{1}, W_{2}, \ldots$ such that $\left(\mathrm{I}_{1}\right)$ and $\left(\mathrm{I}_{2}\right)$ hold for all $n$.

Let $P \in S$. The proof will be complete if we show that $\left\{W_{n}(P) \mid n \in N\right\}$ is a local base at $P$. If there exist infinitely many $n$ such that $W_{n}(P)$ is contained in some set in $B_{n}(P)$, then the conclusion follows since $\left\{\cup B_{n}(P) \mid n \in N\right\}$ is a decreasing local base at $P$. So suppose there is an $m \in N$ such that if $n \geqslant m$, then no set in $B_{n}(P)$ contains $W_{n}(P)$, hence $P \neq x\left(W_{n}(P)\right)$. Thus $\left\{W_{n}(P) \mid n>m\right\}$ is a monotonic subcollection of $B$ and the sequence $\left\{x\left(W_{n}(P)\right)\right\}_{n=m}^{\infty}$ has a cluster point $x$. If $\left\{W_{n}(P) \mid n \geqslant m\right\}$ is not a local base at $P$, then $\bigcap_{n=m}^{\infty} W_{n}(P)$ is open and contains $x$ but contains no $x\left(W_{n}(P)\right)$ for $n \geqslant m$, which is a contradiction. Thus the result follows.

ExAMPLES. As observed in [5], the Michael line is a first countable space having an ortho-base but no base of countable order, so the assumption that $S$ is a monotonic $\beta$-space cannot be omitted from Theorem 3. Also the space $D_{1}^{*}$ (see [5]) is a $\beta$-space having an ortho-base but no base of countable order, hence first countability is also needed.

Question. Does Theorem 3 remain valid if the monotonic conditions are deleted?

\section{REFERENCES}

1. D. K. Burke, $A$ nondevelopable locally compact Hausdorff space with a $G_{\delta}$-diagonal, General Topology and Appl. 2 (1972), 287-291. MR 47 \# 7702.

2. J. Chaber, On point-countable collections and monotonic properties, Fund. Math. (to appear).

3. P. Fletcher and W. F. Lindgren, Orthocompactness and strong Cech completeness in Moore spaces, Duke Math. J. 39 (1972), 753-766. MR 47 \# 1029.

4. R. E. Hodel, Moore spaces and $w \Delta$-spaces, Pacific J. Math. 38 (1971), 641-652. MR 46 \#6290.

5. P. J. Nyikos, Some surprising base properties in topology, Proc. Topology Conf. (Univ. of North Carolina, Charlotte, N. C.), Academic Press, New York, 1974, pp. 427-450. 
6. H. H. Wicke, Open continuous images of certain kinds of $M$-spaces and completeness of mappings and spaces, General Topology and Appl. 1 (1971), 85-100. MR 43 \#8060.

7. H. H. Wicke and J. M. Worrell, Completeness and topologically uniformizing structure, Proc. Second Pittsburgh Internat. Conf. General Topology and Appl. (1972), Springer-Verlag, New York, 1974, pp. 557-585.

8. , Primitive structures in general topology, Proc. Topology Conf. (Univ. of North Carolina, Chariotte, N.C.), Academic Press, New York, 1974, pp. 581-599.

9. Topological completeness of first countable Hausdorff spaces. I, Fund. Math. 75 (1972), 209-222. MR 46 \#8189.

Department of Mathimatics, Auburn Universtty, Auburn, Alabama 36830 